\title{
EVALUATION OF BEETROOT QUALITY DURING VARIOUS STORAGE CONDITIONS
}

\author{
Jonas Viskelis $^{1 *}$, Skirmantas Nevidomskis ${ }^{2}$, Ceslovas Bobinas ${ }^{1}$, Dalia Urbonaviciene ${ }^{1}$, Ramune Bobinaite ${ }^{1}$, \\ Rasa Karkleliene ${ }^{1}$, Pranas Viskelis ${ }^{1,3}$ \\ ${ }^{l}$ Institute of Horticulture, Lithuanian Research Centre for Agriculture and Forestry, Kauno st. 30, Babtai, Kaunas distr., Lithuania, \\ e-mail: j.viskelis@lsdi.lt \\ ${ }^{2}$ Kedainiu konservu fabrikas JSC, Kedainiu str. 50, Singaliai, Kedainiai distr., Lithuania \\ ${ }^{3}$ Department of Food Science and Technology, Kaunas University of Technology, Radvilenu rd. 19, Kaunas, Lithuania
}

\begin{abstract}
To produce high quality processed products, it is necessary to not only grow new, promising beetroot varieties, but also to preserve their quality during storage. This requires advanced storage technologies, such as storage in a controlled and ultra-low oxygen atmosphere. In these conditions, beetroots retain their quality until the new harvest. This not only maximizes the quality of vegetables, reduces storage losses, but also extends the use of vegetables, which is very important for farmers to profitably of their produce. The aim of the work was to evaluate and optimize the process of storing various varieties of beetroot and evaluate their suitability for processing. Beetroot varieties 'Detroit 2', 'Boro H', 'Boltardy', 'Kestrel H',' Pablo H', 'Bona', 'Wodan H', 'Rhonda H', 'Subeto H', 'Action $\mathrm{H}$ ', and 'Joniai' were investigated. Increasing the carbon dioxide content from $0.03 \%$ up to $3 \%, 5 \%$ or $8 \%$ and reducing oxygen content from $21 \%$ to $10 \%$ or $5 \%$ positively impacts the chemical composition of the stored beetroots. Some of the chemical composition parameters after storage in one or another composition in the controlled atmosphere were unchanged, but others remained stable and the degradation of betacyanins was clearly reduced. After the evaluation of various varieties of beetroots after storage, the following varieties were found to be the most suitable for storage and subsequent processing: 'Kestrel H', 'Joniai', 'Pablo H', and 'Rhonda H'. The optimal composition of controlled atmosphere for beetroot storage is 5\% carbon dioxide, 5\% oxygen and $90 \%$ nitrogen.
\end{abstract}

Keywords: beetroot; chemical composition; controlled atmosphere; storage

\section{Introduction}

Beetroots (Beta vulgaris L.) is grown and appreciated for its valuable nutritional properties, easy cultivation and good storage. The considerable advantage of beetroots is that they can be used fresh all year round, they can be stored until the fresh harvest. Beetroots are also very good for processing. Due to its valuable nutritional, dietary properties, beetroot demand and consumption has increased in recent years (Wroblewska et al., 2011; Esatbeyoglu et al., 2015; Moding et al., 2018). Betanine strengthens the walls of vascular capillaries, lowers blood pressure, improves fat metabolism and liver function, protects the cells of our body from the harmful effects of free radicals, as well as cancer, cardiovascular diseases, stops aging processes, also has beneficial effect on health with second-degree diabetes (Gilchrist et al., 2014). The biochemical composition of beetroots is determined by the complex of the genetic characteristics of the variety and the growth conditions, on average they accumulate about $11-17 \%$ of dry matter, $5-10 \%$ of sugars, vitamins C, PP, $\mathrm{B}_{1}, \mathrm{~B}_{2}, \mathrm{~B}_{6}$, lots of potassium (Petronienè, Viškelis, 2004). Beetroots accumulate large amounts of nitrates and may exceed $2000 \mathrm{mg} \mathrm{kg}^{-1}$. The beetroot quality can vary significantly from agrometeorological and growth conditions (Zalatorius et al., 2014).

Storage of beetroot varieties has not been investigated enough. Previous studies have shown that beetroot storage depends on storage conditions, variety, genotype, agroclimatic cultivation conditions, fertilization (Tucker et al., 1977; Henze, Baumann, 1979; Badelek et al., 2002; Bundinienè et al., 2007).

To produce high quality processed products, it is necessary not only to grow new, promising beetroot varieties, but also to preserve their quality during storage. This requires advanced storage technologies, such as storage in a controlled and ultra-low oxygen atmosphere. In these conditions, beetroots retain their quality until the new harvest. This not only maximizes the quality of vegetables, reduces storage losses, but also extends the use of vegetables, which is very important for farmers to profitably of their produce. Most of the studies are long overdue, some beetroot varieties and hybrids have been renewed, it is not clear which varieties with their biochemical composition and technological properties are most suitable for processing, it is unclear whether it is worth storing beetroots in a controlled atmosphere and, if so, under what conditions. Storing vegetables in a controlled atmosphere is universally recognized as a perfect storage technology that allows to store not only most of the production but also preserve biologically active components (Simson, Straus, 2010; Viškelis et al., 2012; Lepse et al., 2014). But for vegetables it is an expensive technology. Somehow there is a belief that it is worth storing only expensive products in a controlled atmosphere, such as berries or fruits, but it is not worth storing cheap vegetables. The aim of the work was to evaluate and optimize the process of storing various varieties of beetroot and evaluate their suitability for processing.

\section{Materials and Methods}

Research object

Beetroots (Beta vulgaris L.) were grown on the test fields of the Institute of Horticulture, Lithuanian Research Centre for Agriculture and Forestry in 2017. The following varieties were tested: 'Detroit 2', 'Boro H', 'Boltardy', 'Kestrel H', 'Pablo H', 'Bona', 'Wodan H', 'Rhonda H', 'Subeto H', 'Action H', and 
'Joniai'. Beetroot chemical composition, physicochemical properties and storage parameters were investigated. Beetroots were cultivated in light loam soil according to the integrated plant cultivation technology adopted by the Institute of Horticulture. Beetroots were harvested in end of September 2017. For analysis, similar sized, raw beetroots were selected.

\section{Quality analysis}

Ascorbic acid was measured by titration using 2,6-dichlorophenolindophenol sodium salt solution $\left(\right.$ AOAC, $1990^{\mathrm{a}}$ ) by a slight modification as by Viskelis et al. (2010).

Soluble solids were quantified with a digital refractometer PR-32 (Atago Co., Ltd., Japan).

Dry matter content was determined gravimetrically by drying samples to a constant weight at $105{ }^{\circ} \mathrm{C}$.

Monosaccharides, sucrose and total sugars were determined by the Bertrand method (AOAC 1990 ${ }^{\mathrm{b}}$ ).

The quantitative and qualitative composition of betalain was determined spectrophotometrically by Wruss et al. (2015) method.

Titratable acidity was estimated by titrating with $0.1 \mathrm{~N} \mathrm{NaOH}$ solution to $\mathrm{pH} 8.0$ and was expressed as a percentage of citric acid equivalent (Ермаков et al., 1987).

Active acidity $(\mathrm{pH})$ was measured with inoLab $\mathrm{pH}$ Level $1 \mathrm{pH}$ meter using SenTix 81 (WTW) electrode.

The amount of nitrates was determined potentiometrically with a selective electrode (Methodological instructions, 1990).

Beetroot colour indexes in the space of even contrast colours were measured with a spectrophotometer MiniScan XE Plus (Hunter Associates Laboratory, Inc., USA) as described in paper of Urbonaviciene et al. (2018). Parameters calculated were chroma (1) and hue angle (2):

$$
\begin{aligned}
& \left(C=\left(a^{* 2}+b^{* 2}\right)^{1 / 2}\right) \\
& \left(h^{\circ}=\arctan \left(\frac{b^{*}}{a^{*}}\right)\right)
\end{aligned}
$$

where: $\mathrm{C}$ - chroma;

$\mathrm{h}^{\circ}$ - hue angle;

$\mathrm{a}^{*}$ - a colour value (redness);

$b^{*}-b$ colour value (yellowness).

Beetroot firmness was determined by the TA.XTPlus texture analyser (Stable Micro Systems, United Kingdom) using the $\mathrm{P} / 2$ probe (2 $\mathrm{mm}$ in diameter) (Luksiene et al., 2013).

\section{Storage}

All beetroot varieties storage has been carried out in three repositories under optimum conditions, at $1 \pm 1{ }^{\circ} \mathrm{C}$ and $90-95 \%$ relative humidity $(\mathrm{RH})$. Selected varieties for best perspectives for processing and storage ('Kestrel H', 'Pablo H', 'Rhonda H' and 'Joniai') were also stored in Besseling CA Systems (Besseling Group B.V., The Netherlands) controlled atmosphere chambers maintaining a different composition of controlled atmosphere (Table 1). Gas composition, humidity, and storage temperature were selected according to the recommended conditions for storing vegetables in a controlled atmosphere (Thompson, 2010; Simson, Straus, 2010; Lepse et al., 2014). The weight of the roots in the controlled atmosphere was accurately weighed and averaged $15 \mathrm{~kg}$. Quality was was studied during 7-month storage.

Table 1

Storage conditions in controlled atmosphere

\begin{tabular}{ccccc}
\hline No. & $\mathbf{C O}_{2}, \boldsymbol{\%}$ & $\mathbf{O}_{2}, \boldsymbol{\%}$ & Temperature, ${ }^{\circ} \mathbf{C}$ & $\mathbf{R H}, \boldsymbol{\%}$ \\
\hline 1 & 0.03 & 21 & $2 \pm 1$ & 95 \\
2 & 3.00 & 10 & $2 \pm 1$ & 95 \\
3 & 5.00 & 10 & $2 \pm 1$ & 95 \\
4 & 8.00 & 10 & $2 \pm 1$ & 95 \\
5 & 5.00 & 5 & $2 \pm 1$ & 95 \\
6 & 0.03 & 21 & $1 \pm 1$ & 95 \\
7 & 0.03 & 21 & $5 \pm 1$ & 95 \\
\hline
\end{tabular}

The statistical methods of data processing

All the experiments were carried out in triplicate. Means and standard deviations were calculated with STATISTICA 10 StatSoft, Inc., USA) and Excel (Microsoft, USA) software. One-way analysis of variance (ANOVA) along with the posthoc Tukey's HSD test was employed for statistical analysis. Differences were considered to be significant at $\mathrm{p}<0.05$.

\section{Results and Discussion}

Changes in dry matter during storage were observed but were not significant (Table 2). After storage, the dry matter content in some beetroot varieties increased, in others decreased. The loss of dry matter occurs due to breathing because it consumes sugars (Viškelienè et al., 2017), but on the other hand, transpiration and water evaporation from the roots are taking place also. That is how two opposing / contradictory physiological processes take place and their relationship is hard to predict. However, in any case, these changes were not statistically reliable. Similar results were obtained with soluble solids (Table 2). During storage, monosaccharide, sucrose and total sugar content varied slightly and results were not statistically significant (Table 2).

Exceptionally high levels of betacyanins and betaxanthin were found in 'Detroit 2' and 'Joniai' beetroots, the highest amount of betalain was in 'Kestrel H' beetroots (Table 3). After storage, the amount of betacyanins and betaxanthin in the 'Detroit 2' and 'Joniai' beetroots decreased significantly, but still the highest amount of betalains remained in these beetroots, $1278 \mathrm{mg} \mathrm{kg}^{-1}$ and $1326 \mathrm{mg} \mathrm{kg}^{-1}$, respectively.

The amount of organic acids during storage was reduced or did not change compared to fresh beetroots of the same variety (Table 3 ). These changes in most varieties were statistically unreliable due to the very low acidity of beetroots.

Statistically reliable post-storage decrease in organic acids was observed only in 'Boltardy', 'Rhonda H' and 'Subeto H' varieties (from $0.10 \%$ to $0.08 \%)(\mathrm{p}<0.05)$. 
Table 2

Changes in dry matter, soluble solids, monosaccharides, sucrose and total sugars during beetroot storage

\begin{tabular}{|c|c|c|c|c|c|c|c|c|c|c|}
\hline \multirow[b]{2}{*}{$\begin{array}{l}\text { Beet } \\
\text { variety }\end{array}$} & \multicolumn{2}{|c|}{ Dry matter, \% } & \multicolumn{2}{|c|}{ Soluble solids, \% } & \multicolumn{2}{|c|}{ Monosaccharides, \% } & \multicolumn{2}{|c|}{ Sucrose, \% } & \multicolumn{2}{|c|}{ Total sugars, \% } \\
\hline & $\begin{array}{c}\text { Before } \\
\text { storage }\end{array}$ & $\begin{array}{c}\text { After } \\
\text { storage }\end{array}$ & $\begin{array}{c}\text { Before } \\
\text { storage }\end{array}$ & $\begin{array}{c}\text { After } \\
\text { storage }\end{array}$ & $\begin{array}{l}\text { Before } \\
\text { storage }\end{array}$ & $\begin{array}{c}\text { After } \\
\text { storage }\end{array}$ & $\begin{array}{c}\text { Before } \\
\text { storage }\end{array}$ & $\begin{array}{c}\text { After } \\
\text { storage }\end{array}$ & $\begin{array}{c}\text { Before } \\
\text { storage }\end{array}$ & $\begin{array}{c}\text { After } \\
\text { storage }\end{array}$ \\
\hline Detroit 2 & $17.1^{\mathrm{a}}$ & $15.9^{\mathrm{a}}$ & $13.6^{\mathrm{a}}$ & $12.6^{\mathrm{a}}$ & $1.41^{\mathrm{a}}$ & $1.31^{\mathrm{a}}$ & $8.23^{\mathrm{a}}$ & $8.34^{\mathrm{a}}$ & $9.64^{\mathrm{a}}$ & $9.65^{\mathrm{a}}$ \\
\hline Boro $\mathrm{H}$ & $13.7^{\mathrm{a}}$ & $14.6^{\mathrm{a}}$ & $11.2^{\mathrm{a}}$ & $11.5^{\mathrm{a}}$ & $1.16^{\mathrm{a}}$ & $1.09^{\mathrm{a}}$ & $8.07^{\mathrm{a}}$ & $8.18^{\mathrm{a}}$ & $9.23^{\mathrm{a}}$ & $9.27^{\mathrm{a}}$ \\
\hline Boltardy & $16.0^{\mathrm{a}}$ & $17.2^{\mathrm{a}}$ & $14.0^{\mathrm{a}}$ & $13.9^{\mathrm{a}}$ & $1.48^{\mathrm{a}}$ & $1.35^{\mathrm{a}}$ & $8.30^{\mathrm{a}}$ & $8.40^{\mathrm{a}}$ & $9.78^{\mathrm{a}}$ & $9.75^{\mathrm{a}}$ \\
\hline Kestrel H & $15.4^{\mathrm{a}}$ & $15.9^{\mathrm{a}}$ & $14.0^{\mathrm{a}}$ & $14.0^{\mathrm{a}}$ & $1.41^{\mathrm{a}}$ & $1.35^{\mathrm{a}}$ & $9.12^{\mathrm{a}}$ & $9.22^{\mathrm{a}}$ & $10.53^{a}$ & $10.57^{\mathrm{a}}$ \\
\hline Pablo H & $13.4^{\mathrm{a}}$ & $14.1^{\mathrm{a}}$ & $12.0^{\mathrm{a}}$ & $12.9^{\mathrm{a}}$ & $1.54^{\mathrm{a}}$ & $1.44^{\mathrm{a}}$ & $8.89^{\mathrm{a}}$ & $8.94^{\mathrm{a}}$ & $10.43^{\mathrm{a}}$ & $10.38^{\mathrm{a}}$ \\
\hline Bona & $15.9^{\mathrm{a}}$ & $14.5^{\mathrm{a}}$ & $13.8^{\mathrm{a}}$ & $11.8^{\mathrm{a}}$ & $1.22^{\mathrm{a}}$ & $1.22^{\mathrm{a}}$ & $7.77^{\mathrm{a}}$ & $7.85^{\mathrm{a}}$ & $8.99^{\mathrm{a}}$ & $9.07^{\mathrm{a}}$ \\
\hline Wodan $\mathrm{H}$ & $13.0^{\mathrm{a}}$ & $14.1^{\mathrm{a}}$ & $11.7^{\mathrm{a}}$ & $12.0^{\mathrm{a}}$ & $1.22^{\mathrm{a}}$ & $1.22^{\mathrm{a}}$ & $8.79^{\mathrm{a}}$ & $8.22^{\mathrm{a}}$ & $10.01^{\mathrm{a}}$ & $9.44^{\mathrm{a}}$ \\
\hline Rhonda H & $15.1^{\mathrm{a}}$ & $14.0^{\mathrm{a}}$ & $12.0^{\mathrm{a}}$ & $12.8^{\mathrm{a}}$ & $1.22^{\mathrm{a}}$ & $1.32^{\mathrm{a}}$ & $8.29^{\mathrm{a}}$ & $8.09^{\mathrm{a}}$ & $9.51^{\mathrm{a}}$ & $9.41^{\mathrm{a}}$ \\
\hline Subeto H & $13.1^{\mathrm{a}}$ & $13.9^{\mathrm{a}}$ & $11.0^{\mathrm{a}}$ & $11.5^{\mathrm{a}}$ & $1.35^{\mathrm{a}}$ & $1.22^{\mathrm{a}}$ & $8.29^{\mathrm{a}}$ & $8.09^{\mathrm{a}}$ & $9.64^{\mathrm{a}}$ & $9.31^{\mathrm{a}}$ \\
\hline Action $\mathrm{H}$ & $13.8^{\mathrm{a}}$ & $14.7^{\mathrm{a}}$ & $12.0^{\mathrm{a}}$ & $12.6^{\mathrm{a}}$ & $1.09^{\mathrm{a}}$ & $1.12^{\mathrm{a}}$ & $8.14^{\mathrm{a}}$ & $8.01^{\mathrm{a}}$ & $9.23^{\mathrm{a}}$ & $9.13^{\mathrm{a}}$ \\
\hline Joniai & $14.8^{\mathrm{a}}$ & $13.6^{\mathrm{a}}$ & $13.1^{\mathrm{a}}$ & $12.5^{\mathrm{a}}$ & $1.28^{\mathrm{a}}$ & $1.34^{\mathrm{a}}$ & $8.87^{\mathrm{a}}$ & $8.75^{\mathrm{a}}$ & $10.15^{\mathrm{a}}$ & $10.09^{\mathrm{a}}$ \\
\hline
\end{tabular}

Different letters in "before storage" and "after storage" columns represent statistically significant differences in chemical composition parameters before and after storage.

Table 3

Changes in betacyanin, betaxanthin, acidity, pH and ascorbic acid content during beetroot storage

\begin{tabular}{|c|c|c|c|c|c|c|c|c|c|c|}
\hline \multirow{2}{*}{$\begin{array}{l}\text { Beet } \\
\text { variety }\end{array}$} & \multicolumn{2}{|c|}{$\begin{array}{c}\text { Betacyanins, } \\
\mathrm{mg} \mathrm{kg}^{-1}\end{array}$} & \multicolumn{2}{|c|}{$\begin{array}{l}\text { Betaxanthins, } \\
\text { mg kg-1 }^{-1}\end{array}$} & \multicolumn{2}{|c|}{ Acidity, \% } & \multicolumn{2}{|c|}{$\mathbf{p H}$} & \multicolumn{2}{|c|}{$\begin{array}{l}\text { Ascorbic acid, } \\
\text { mg } 100 \mathrm{~g}^{-1}\end{array}$} \\
\hline & $\begin{array}{c}\text { Before } \\
\text { storage }\end{array}$ & $\begin{array}{c}\text { After } \\
\text { storage }\end{array}$ & $\begin{array}{c}\text { Before } \\
\text { storage }\end{array}$ & $\begin{array}{c}\text { After } \\
\text { storage }\end{array}$ & $\begin{array}{c}\text { Before } \\
\text { storage }\end{array}$ & $\begin{array}{c}\text { After } \\
\text { storage }\end{array}$ & $\begin{array}{c}\text { Before } \\
\text { storage }\end{array}$ & $\begin{array}{c}\text { After } \\
\text { storage }\end{array}$ & $\begin{array}{c}\text { Before } \\
\text { storage }\end{array}$ & $\begin{array}{c}\text { After } \\
\text { storage }\end{array}$ \\
\hline Detroit 2 & $967^{\mathrm{a}}$ & $883^{a}$ & $423^{a}$ & $395^{\mathrm{a}}$ & $0.09^{a}$ & $0.08^{\mathrm{a}}$ & $5.70^{\mathrm{a}}$ & $5.75^{\mathrm{a}}$ & $17.6^{\mathrm{a}}$ & $15.2^{\mathrm{b}}$ \\
\hline Boro H & $474^{b}$ & $589^{a}$ & $259^{a}$ & $275^{\mathrm{a}}$ & $0.08^{a}$ & $0.07^{\mathrm{a}}$ & $5.78^{a}$ & $5.78^{a}$ & $16.8^{a}$ & $14.0^{\mathrm{b}}$ \\
\hline Boltardy & $562^{b}$ & $671^{a}$ & $271^{\mathrm{b}}$ & $347^{\mathrm{a}}$ & $0.10^{\mathrm{a}}$ & $0.08^{b}$ & $5.70^{\mathrm{a}}$ & $5.77^{\mathrm{a}}$ & $16.1^{\mathrm{a}}$ & $13.2^{\mathrm{b}}$ \\
\hline Kestrel H & $642^{a}$ & $695^{a}$ & $363^{a}$ & $324^{\mathrm{a}}$ & $0.09^{a}$ & $0.09^{a}$ & $5.77^{\mathrm{a}}$ & $5.75^{a}$ & $19.5^{\mathrm{a}}$ & $16.8^{b}$ \\
\hline Pablo H & $397^{b}$ & $584^{\mathrm{a}}$ & $231^{\mathrm{b}}$ & $301^{\mathrm{a}}$ & $0.08^{a}$ & $0.08^{a}$ & $5.78^{a}$ & $5.79^{a}$ & $18.2^{\mathrm{a}}$ & $15.9^{b}$ \\
\hline Bona & $589^{a}$ & $648^{a}$ & $309^{b}$ & $371^{\mathrm{a}}$ & $0.08^{a}$ & $0.07^{\mathrm{a}}$ & $5.72^{a}$ & $5.74^{\mathrm{a}}$ & $15.8^{\mathrm{a}}$ & $13.5^{b}$ \\
\hline Wodan $\mathrm{H}$ & $595^{\mathrm{a}}$ & $674^{a}$ & $327^{b}$ & $401^{\mathrm{a}}$ & $0.09^{a}$ & $0.08^{a}$ & $5.72^{a}$ & $5.74^{\mathrm{a}}$ & $16.4^{\mathrm{a}}$ & $12.2^{\mathrm{b}}$ \\
\hline Rhonda $\mathrm{H}$ & $505^{\mathrm{b}}$ & $681^{a}$ & $300^{\mathrm{a}}$ & $344^{\mathrm{a}}$ & $0.10^{\mathrm{a}}$ & $0.08^{b}$ & $5.75^{a}$ & $5.76^{\mathrm{a}}$ & $18.3^{\mathrm{a}}$ & $15.7^{b}$ \\
\hline Subeto H & $403^{b}$ & $592^{\mathrm{a}}$ & $258^{\mathrm{a}}$ & $251^{\mathrm{a}}$ & $0.10^{\mathrm{a}}$ & $0.08^{b}$ & $5.71^{\mathrm{a}}$ & $5.74^{\mathrm{a}}$ & $13.2^{\mathrm{a}}$ & $10.4^{b}$ \\
\hline Action $\mathrm{H}$ & $412^{b}$ & $528^{a}$ & $246^{\mathrm{b}}$ & $311^{\mathrm{a}}$ & $0.09^{a}$ & $0.09^{\mathrm{a}}$ & $5.70^{\mathrm{a}}$ & $5.74^{\mathrm{a}}$ & $13.4^{\mathrm{a}}$ & $10.8^{\mathrm{b}}$ \\
\hline Joniai & $1030^{a}$ & $887^{\mathrm{b}}$ & $608^{a}$ & $439^{b}$ & $0.09^{a}$ & $0.08^{a}$ & $5.71^{\mathrm{a}}$ & $5.74^{\mathrm{a}}$ & $17.8^{\mathrm{a}}$ & $15.6^{b}$ \\
\hline
\end{tabular}

Different letters in "before storage" and "after storage" columns for each parameter represent statistically significant differences in chemical composition parameters before and after storage.

Table 4

Changes in nitrates, skin and flesh firmness and colour indices during beetroot storage

\begin{tabular}{|c|c|c|c|c|c|c|c|c|c|c|}
\hline \multirow{2}{*}{$\begin{array}{l}\text { Beet } \\
\text { variety }\end{array}$} & \multicolumn{2}{|c|}{$\begin{array}{l}\text { Nitrates, } \\
\text { mg kg-1 }^{-1}\end{array}$} & \multicolumn{2}{|c|}{$\begin{array}{l}\text { Skin firmness, } \\
\quad \mathrm{N} \mathrm{cm}^{-2}\end{array}$} & \multicolumn{2}{|c|}{$\begin{array}{l}\text { Flesh firmness, } \\
\quad \mathrm{N} \mathrm{cm}^{-2}\end{array}$} & \multicolumn{2}{|c|}{ Colour a* value } & \multicolumn{2}{|c|}{ Colour $b^{*}$ value } \\
\hline & $\begin{array}{c}\text { Before } \\
\text { storage }\end{array}$ & $\begin{array}{c}\text { After } \\
\text { storage }\end{array}$ & $\begin{array}{c}\text { Before } \\
\text { storage }\end{array}$ & $\begin{array}{c}\text { After } \\
\text { storage }\end{array}$ & $\begin{array}{l}\text { Before } \\
\text { storage }\end{array}$ & $\begin{array}{c}\text { After } \\
\text { storage }\end{array}$ & $\begin{array}{c}\text { Before } \\
\text { storage }\end{array}$ & $\begin{array}{c}\text { After } \\
\text { storage }\end{array}$ & $\begin{array}{l}\text { Before } \\
\text { storage }\end{array}$ & $\begin{array}{c}\text { After } \\
\text { storage }\end{array}$ \\
\hline Detroit 2 & $805^{\mathrm{a}}$ & $758^{a}$ & $735^{a}$ & $648^{b}$ & $446^{\mathrm{a}}$ & $392^{b}$ & $7.07^{\mathrm{a}}$ & $5.16^{\mathrm{b}}$ & $0.97^{\mathrm{b}}$ & $1.49^{\mathrm{a}}$ \\
\hline Boro $\mathrm{H}$ & $717^{\mathrm{a}}$ & $705^{\mathrm{a}}$ & $549^{a}$ & $532^{\mathrm{a}}$ & $338^{\mathrm{a}}$ & $322^{\mathrm{a}}$ & $8.41^{\mathrm{a}}$ & $7.21^{\mathrm{b}}$ & $1.61^{\mathrm{a}}$ & $1.74^{\mathrm{a}}$ \\
\hline Boltardy & $751^{\mathrm{a}}$ & $722^{a}$ & $597^{\mathrm{a}}$ & $520^{b}$ & $333^{\mathrm{a}}$ & $308^{a}$ & $7.77^{\mathrm{a}}$ & $7.89^{\mathrm{a}}$ & $1.22^{\mathrm{b}}$ & $1.85^{\mathrm{a}}$ \\
\hline Kestrel H & $685^{a}$ & $589^{\mathrm{b}}$ & $623^{a}$ & $612^{\mathrm{a}}$ & $401^{\mathrm{a}}$ & $388^{\text {a }}$ & $6.63^{b}$ & $9.92^{\mathrm{a}}$ & $1.20^{\mathrm{a}}$ & $1.29^{\mathrm{a}}$ \\
\hline Pablo H & $751^{\mathrm{a}}$ & $623^{\mathrm{b}}$ & $529^{a}$ & $532^{\mathrm{a}}$ & $337^{\text {a }}$ & $339^{a}$ & $5.99^{b}$ & $8.18^{\mathrm{a}}$ & $1.09^{b}$ & $1.88^{\mathrm{a}}$ \\
\hline Bona & $701^{a}$ & $602^{\mathrm{b}}$ & $625^{a}$ & $584^{\mathrm{a}}$ & $401^{\mathrm{a}}$ & $384^{\mathrm{a}}$ & $6.08^{\mathrm{a}}$ & $6.26^{\mathrm{a}}$ & $0.80^{\mathrm{b}}$ & $1.52^{\mathrm{a}}$ \\
\hline Wodan H & $701^{a}$ & $741^{\mathrm{a}}$ & $594^{\mathrm{a}}$ & $578^{a}$ & $355^{\mathrm{a}}$ & $275^{b}$ & $6.47^{\mathrm{a}}$ & $6.67^{\mathrm{a}}$ & $1.01^{\mathrm{b}}$ & $2.12^{\mathrm{a}}$ \\
\hline Rhonda H & $701^{\mathrm{a}}$ & $680^{\mathrm{a}}$ & $663^{\mathrm{a}}$ & $655^{\mathrm{a}}$ & $416^{\mathrm{a}}$ & $394^{\mathrm{a}}$ & $9.41^{\mathrm{a}}$ & $8.25^{\mathrm{b}}$ & $1.24^{\mathrm{b}}$ & $2.33^{\mathrm{a}}$ \\
\hline Subeto H & $734^{\mathrm{a}}$ & $771^{\mathrm{a}}$ & $548^{a}$ & $551^{\text {a }}$ & $354^{\mathrm{a}}$ & $348^{\text {a }}$ & $6.42^{b}$ & $10.91^{\mathrm{a}}$ & $0.94^{\mathrm{b}}$ & $2.10^{\mathrm{a}}$ \\
\hline Action $\mathrm{H}$ & $769^{\mathrm{a}}$ & $797^{\mathrm{a}}$ & $567^{\mathrm{a}}$ & $551^{\mathrm{a}}$ & $357^{\mathrm{a}}$ & $326^{\mathrm{a}}$ & $4.90^{\mathrm{b}}$ & $10.71^{\mathrm{a}}$ & $0.52^{b}$ & $2.93^{\mathrm{a}}$ \\
\hline Joniai & $670^{\mathrm{a}}$ & $642^{\mathrm{a}}$ & $766^{\mathrm{a}}$ & $611^{\mathrm{b}}$ & $478^{a}$ & $385^{\mathrm{b}}$ & $8.62^{\mathrm{a}}$ & $7.33^{b}$ & $1.39^{\mathrm{b}}$ & $1.80^{\mathrm{a}}$ \\
\hline
\end{tabular}

Different letters in "before storage" and "after storage" columns for each parameter represent statistically significant differences in beetroot quality parameters before and after storage.

During storage, $\mathrm{pH}$ tended to increase, but the results of the $\mathrm{pH}$ increase were statistically insignificant (Table 3 ). The amount of ascorbic acid in beetroots decreased statistically reliably (Table 3). Average loss of vitamin C during storage was about $15-20 \%$ from its original content in fresh beetroots. After 7 months of storage, the least ascorbic acid decrease was observed in 'Kestrel H', 'Pablo H', 'Rhonda H' and 'Joniai' varieties. Changes in nitrate content in the stored beetroots was not statistically significant in most varieties, except in ''Kestrel H', 'Pablo H', and 'Bona' varieties (Table 4). 
Colour measurement also showed degradation of some compounds during storage. Beetroots of all varieties tested darkened during storage, i.e., their brightness coordinate $\mathrm{L}^{*}$ decreased. The redness coordinate $\left(\mathrm{a}^{*}\right)$ in most cases increased, especially in 'Subeto $H^{\prime}$ ', 'Action H', 'Kestrel H', 'Pablo H' varieties. However, the yellowness value $\left(b^{*}\right)$ increased significantly during storage in all varieties (Table 4). This indicates that biochemical and certain degradation processes occur during the storage of beetroots. In the case of beetroot storage, the colour purity ( $\mathrm{C}^{*}$, chroma) varied unevenly: in some varieties it decreased, but in the majority of varieties increased. After storage, 'Subeto H', 'Action H', 'Kestrel H', 'Pablo H' varieties had the purest colour.

After 7 months of storage, the firmness of the beetroots reduced for both the skin and flesh (Table 4). The decrease in the firmness of both skin and flesh can be explained by the fact that beetroots still mature and age, hydrolysis of insoluble protopectin to soluble pectin takes place etc. The most important indicator for storage is the percentage of production after storage. The average production of beetroots was $67 \%$ after storage, hence, storage losses averaged $33 \%$, similar as storage of onions (Cizauskas, Viskelis, 2002). The beetroots of
'Kestrel H' variety remained up to $78 \%$. Other good varieties for storage were 'Pablo H', 'Bona', 'Subeto H' and 'Joniai'. Although storage losses seem great, but stored beetroots were quite acceptable. Storage loss consists of natural loss of mass due to respiration, transpiration and rotting. After storage the beetroot ringing had a clear tendency to decrease. During storage, due to the decrease in the firmness, betalains diffused into the lighter layers of the flesh, so in the overall assessment the internal ringing were increased. The 'Joniai' beetroots were the only one of the studied varieties to have no ringing after storage. After the total evaluation of the various varieties of beetroots after storage, the following varieties were found to be the most suitable for storage and processing: 'Kestrel H', 'Joniai', 'Pablo H' and 'Rhonda H'.

Changes in chemical composition and physical properties such as firmness, colour, etc. during storage are determined not only by the genotype of the variety, but also by the agrotechnical tools used (Petronienè, Viškelis, 2004; Bundinienè et al., 2007).

Beetroot is susceptible to boron deficiency, and the use of boron fertilizers not only increases yield but also reduces storage losses due to physiological damage related to the lack of boron (Bundiniene et al., 2017).

Table 5

Changes in betacyanin, betaxanthin, nitrates, flesh firmness and redness colour in beetroots during storage in controlled atmosphere chambers

\begin{tabular}{|c|c|c|c|c|c|c|c|c|c|c|c|}
\hline \multirow{2}{*}{ Variant* } & \multirow{2}{*}{$\begin{array}{c}\text { Beet } \\
\text { variety }\end{array}$} & \multicolumn{2}{|c|}{$\begin{array}{c}\text { Betacyanins, } \\
\text { mg kg-1 }^{-1}\end{array}$} & \multicolumn{2}{|c|}{$\begin{array}{c}\text { Betaxanthins, } \\
\text { mg kg }^{-1}\end{array}$} & \multicolumn{2}{|c|}{$\begin{array}{l}\text { Nitrates, } \\
\text { mg kg }^{-1}\end{array}$} & \multicolumn{2}{|c|}{$\begin{array}{l}\text { Flesh firmness, } \\
\mathrm{N} \mathrm{cm}^{-2}\end{array}$} & \multicolumn{2}{|c|}{ Colour a* value } \\
\hline & & $\begin{array}{l}\text { Before } \\
\text { storage }\end{array}$ & $\begin{array}{c}\text { After } \\
\text { storage }\end{array}$ & $\begin{array}{l}\text { Before } \\
\text { storage }\end{array}$ & $\begin{array}{c}\text { After } \\
\text { storage }\end{array}$ & $\begin{array}{l}\text { Before } \\
\text { storage }\end{array}$ & $\begin{array}{c}\text { After } \\
\text { storage }\end{array}$ & $\begin{array}{l}\text { Before } \\
\text { storage }\end{array}$ & $\begin{array}{c}\text { After } \\
\text { storage }\end{array}$ & $\begin{array}{l}\text { Before } \\
\text { storage }\end{array}$ & $\begin{array}{c}\text { After } \\
\text { storage }\end{array}$ \\
\hline \multirow[t]{4}{*}{1} & Kestrel H & $643^{\mathrm{a}}$ & $678^{a}$ & $364^{\mathrm{a}}$ & $328^{\mathrm{b}}$ & $685^{\mathrm{a}}$ & $584^{\mathrm{b}}$ & $401^{\mathrm{a}}$ & $375^{\mathrm{a}}$ & $6.63^{\mathrm{b}}$ & $8.97^{\mathrm{a}}$ \\
\hline & Pablo H & $397^{b}$ & $574^{\mathrm{a}}$ & $232^{b}$ & $298^{\mathrm{a}}$ & $751^{a}$ & $611^{\mathrm{b}}$ & $337^{\mathrm{a}}$ & $324^{\mathrm{a}}$ & $5.99^{\mathrm{b}}$ & $7.24^{\mathrm{a}}$ \\
\hline & Rhonda H & $505^{b}$ & $687^{\mathrm{a}}$ & $300^{a}$ & $321^{\mathrm{a}}$ & $701^{\mathrm{a}}$ & $644^{\mathrm{a}}$ & $416^{\mathrm{a}}$ & $384^{\mathrm{a}}$ & $9.41^{\mathrm{a}}$ & $8.35^{\mathrm{b}}$ \\
\hline & Joniai & $1031^{\mathrm{a}}$ & $866^{\mathrm{b}}$ & $608^{a}$ & $558^{\mathrm{a}}$ & $670^{\mathrm{a}}$ & $621^{\mathrm{a}}$ & $478^{\mathrm{a}}$ & $359^{\mathrm{b}}$ & $8.62^{\mathrm{a}}$ & $7.66^{\mathrm{b}}$ \\
\hline \multirow[t]{4}{*}{2} & Kestrel H & $643^{a}$ & $655^{\mathrm{a}}$ & $364^{\mathrm{a}}$ & $378^{a}$ & $685^{\mathrm{a}}$ & $579^{b}$ & $401^{\mathrm{a}}$ & $395^{a}$ & $6.63^{b}$ & $7.87^{\mathrm{a}}$ \\
\hline & Pablo H & $397^{\mathrm{a}}$ & $324^{\mathrm{b}}$ & $232^{\mathrm{b}}$ & $378^{\mathrm{a}}$ & $751^{\mathrm{a}}$ & $688^{\mathrm{a}}$ & $337^{\mathrm{a}}$ & $331^{\mathrm{a}}$ & $5.99^{\mathrm{a}}$ & $6.19^{\mathrm{a}}$ \\
\hline & Rhonda H & $505^{b}$ & $621^{\mathrm{a}}$ & $300^{a}$ & $304^{\mathrm{a}}$ & $701^{\mathrm{a}}$ & $624^{\mathrm{b}}$ & $416^{\mathrm{a}}$ & $395^{\mathrm{a}}$ & $9.41^{\mathrm{a}}$ & $8.99^{\mathrm{a}}$ \\
\hline & Joniai & $1031^{\mathrm{a}}$ & $977^{\text {a }}$ & $608^{a}$ & $558^{\mathrm{a}}$ & $670^{a}$ & $655^{\mathrm{a}}$ & $478^{\mathrm{a}}$ & $395^{b}$ & $8.62^{\mathrm{a}}$ & $8.33^{\mathrm{a}}$ \\
\hline \multirow[t]{4}{*}{3} & Kestrel H & $643^{a}$ & $635^{\mathrm{a}}$ & $364^{\mathrm{a}}$ & $354^{\mathrm{a}}$ & $685^{a}$ & $644^{\mathrm{a}}$ & $401^{\mathrm{a}}$ & $391^{\mathrm{a}}$ & $6.63^{b}$ & $8.92^{\mathrm{a}}$ \\
\hline & Pablo H & $397^{\mathrm{b}}$ & $484^{\mathrm{a}}$ & $232^{\mathrm{b}}$ & $322^{\mathrm{a}}$ & $751^{a}$ & $711^{\mathrm{a}}$ & $337^{\mathrm{a}}$ & $321^{\mathrm{a}}$ & $5.99^{\mathrm{a}}$ & $6.18^{\mathrm{a}}$ \\
\hline & Rhonda H & $505^{b}$ & $601^{\mathrm{a}}$ & $300^{\mathrm{a}}$ & $304^{\mathrm{a}}$ & $701^{\mathrm{a}}$ & $688^{a}$ & $416^{\mathrm{a}}$ & $402^{a}$ & $9.41^{\mathrm{a}}$ & $9.25^{\mathrm{a}}$ \\
\hline & Joniai & $1031^{\mathrm{a}}$ & $924^{b}$ & $608^{a}$ & $589^{a}$ & $670^{a}$ & $649^{a}$ & $478^{\mathrm{a}}$ & $426^{\mathrm{b}}$ & $8.62^{\mathrm{a}}$ & $8.35^{\mathrm{a}}$ \\
\hline \multirow[t]{4}{*}{4} & Kestrel H & $643^{a}$ & $652^{a}$ & $364^{a}$ & $358^{a}$ & $685^{a}$ & $642^{a}$ & $401^{a}$ & $396^{\mathrm{a}}$ & $6.63^{a}$ & $6.91^{\mathrm{a}}$ \\
\hline & Pablo H & $397^{\mathrm{a}}$ & $414^{\mathrm{a}}$ & $232^{a}$ & $244^{\mathrm{a}}$ & $751^{a}$ & $678^{\mathrm{b}}$ & $337^{\mathrm{a}}$ & $339^{a}$ & $5.99^{\mathrm{a}}$ & $6.18^{\mathrm{a}}$ \\
\hline & Rhonda H & $505^{b}$ & $642^{\mathrm{a}}$ & $300^{a}$ & $289^{a}$ & $701^{\mathrm{a}}$ & $689^{a}$ & $416^{\mathrm{a}}$ & $421^{a}$ & $9.41^{\mathrm{a}}$ & $9.38^{\mathrm{a}}$ \\
\hline & Joniai & $1031^{\mathrm{a}}$ & $977^{\text {a }}$ & $608^{a}$ & $587^{\mathrm{a}}$ & $670^{a}$ & $655^{\mathrm{a}}$ & $478^{a}$ & $447^{\mathrm{a}}$ & $8.62^{\mathrm{a}}$ & $7.98^{\mathrm{a}}$ \\
\hline \multirow[t]{4}{*}{5} & Kestrel H & $643^{a}$ & $681^{\mathrm{a}}$ & $364^{a}$ & $351^{\mathrm{a}}$ & $685^{\mathrm{a}}$ & $587^{b}$ & $401^{a}$ & $405^{\mathrm{a}}$ & $6.63^{a}$ & $6.85^{\mathrm{a}}$ \\
\hline & Pablo H & $397^{\mathrm{a}}$ & $391^{a}$ & $232^{\mathrm{a}}$ & $244^{\mathrm{a}}$ & $751^{a}$ & $713^{\mathrm{a}}$ & $337^{\mathrm{a}}$ & $322^{\mathrm{a}}$ & $5.99^{\mathrm{a}}$ & $6.25^{\mathrm{a}}$ \\
\hline & Rhonda H & $505^{\mathrm{a}}$ & $491^{\mathrm{a}}$ & $300^{a}$ & $311^{\mathrm{a}}$ & $701^{\mathrm{a}}$ & $710^{\mathrm{a}}$ & $416^{\mathrm{a}}$ & $399^{a}$ & $9.41^{\mathrm{a}}$ & $9.11^{\mathrm{a}}$ \\
\hline & Joniai & $1031^{\mathrm{a}}$ & $977^{\text {a }}$ & $608^{a}$ & $588^{\mathrm{a}}$ & $670^{a}$ & $688^{a}$ & $478^{\mathrm{a}}$ & $459^{a}$ & $8.62^{\mathrm{a}}$ & $8.58^{\mathrm{a}}$ \\
\hline \multirow[t]{4}{*}{6} & Kestrel H & $643^{a}$ & $654^{\mathrm{a}}$ & $364^{a}$ & $333^{a}$ & $685^{a}$ & $628^{a}$ & $401^{\mathrm{a}}$ & $389^{a}$ & $6.63^{b}$ & $8.12^{\mathrm{a}}$ \\
\hline & Pablo H & $397^{\mathrm{a}}$ & $441^{\mathrm{a}}$ & $232^{\mathrm{b}}$ & $288^{a}$ & $751^{a}$ & $623^{b}$ & $337^{\mathrm{a}}$ & $328^{\mathrm{a}}$ & $5.99^{b}$ & $7.18^{\mathrm{a}}$ \\
\hline & Rhonda H & $505^{\mathrm{a}}$ & $521^{\mathrm{a}}$ & $300^{\mathrm{b}}$ & $352^{\mathrm{a}}$ & $701^{a}$ & $694^{\mathrm{a}}$ & $416^{\mathrm{a}}$ & $391^{\mathrm{a}}$ & $9.41^{\mathrm{a}}$ & $8.67^{\mathrm{a}}$ \\
\hline & Joniai & $1031^{\mathrm{a}}$ & $977^{\mathrm{a}}$ & $608^{a}$ & $576^{\mathrm{a}}$ & $670^{a}$ & $652^{\mathrm{a}}$ & $478^{\mathrm{a}}$ & $378^{\mathrm{b}}$ & $8.62^{\mathrm{a}}$ & $7.98^{\mathrm{a}}$ \\
\hline \multirow[t]{4}{*}{7} & Kestrel H & $643^{b}$ & $721^{a}$ & $364^{a}$ & $387^{\mathrm{a}}$ & $685^{a}$ & $541^{\mathrm{b}}$ & $401^{\mathrm{a}}$ & $358^{\mathrm{b}}$ & $6.63^{b}$ & $9.98^{\mathrm{a}}$ \\
\hline & Pablo H & $397^{b}$ & $598^{\mathrm{a}}$ & $232^{\mathrm{b}}$ & $322^{a}$ & $751^{a}$ & $601^{\mathrm{b}}$ & $337^{\mathrm{a}}$ & $301^{\mathrm{b}}$ & $5.99^{\mathrm{b}}$ & $8.48^{\mathrm{a}}$ \\
\hline & Rhonda H & $505^{b}$ & $699^{a}$ & $300^{\mathrm{b}}$ & $358^{\mathrm{a}}$ & $701^{\mathrm{a}}$ & $621^{\mathrm{b}}$ & $416^{\mathrm{a}}$ & $358^{\mathrm{b}}$ & $9.41^{\mathrm{a}}$ & $7.25^{\mathrm{b}}$ \\
\hline & Joniai & $1031^{\mathrm{a}}$ & $799^{b}$ & $608^{a}$ & $421^{b}$ & $670^{a}$ & $611^{\mathrm{a}}$ & $478^{\mathrm{a}}$ & $342^{b}$ & $8.62^{\mathrm{a}}$ & $7.01^{b}$ \\
\hline
\end{tabular}

Different letters in "before storage" and "after storage" columns for each parameter represent statistically significant differences in beetroot quality parameters before and after storage.

* Storage conditions are given in Table 1 
Beetroots were stored not only under optimal conditions, i.e., at $+1 \pm 1^{\circ} \mathrm{C}$ and $90-95 \%$ relative humidity, but also selected varieties for best perspectives for processing and storage ('Kestrel $\mathrm{H}$ ', 'Pablo H', 'Rhonda H' and 'Joniai') were also stored in Besseling CA Systems controlled atmosphere chambers maintaining a different composition of controlled atmosphere (Table 1). During storage, the controlled atmosphere chambers were monitored not only for temperature, humidity, oxygen and carbon dioxide concentrations, but also for the amount of endogenous ripening and aging hormone ethylene. Ethylene was permanently removed catalytically.

While analysing changes in chemical composition during storage (Table 5), it can be concluded that increasing the carbon dioxide content from $0.03 \%$ up to $3 \%, 5 \%$ or $8 \%$ and reduced oxygen content from $21 \%$ to $10 \%$ or $5 \%$ positively impacts chemical composition of the stored beetroots. Some of the chemical composition parameters after storage in one or another composition in the controlled atmosphere remained stable and the degradation of betacyanins was clearly reduced. Beetroots kept in a controlled atmosphere retained a more stable colour, had lower colour changes after storage compared to fresh beetroots. Similarly, beetroot firmness remained closer to the firmness of the fresh beetroots.

From the results of chemical composition, colour coordinates and texture (Table 5), it can be concluded that the best regime to store the beetroots is in atmosphere containing $5 \%$ of carbon dioxide and $5 \%$ of oxygen. At higher oxygen levels, both chemical and physical indicators suffer a little more during storage. This optimal composition in the controlled atmosphere increases around $10 \%$ of standard production compared to storage in simple storage chambers. Beetroot 'Joniai' which was rated very well by its chemical composition and physical properties, while stored in basic storage, they kept only moderately well, but it was excellent in a controlled atmosphere, with storage losses of just $24 \%$.

\section{Conclusions}

After examining the chemical composition, texture and colour coordinates of various varieties of beetroots, the optimal varieties for processing are 'Joniai', 'Rhonda H', 'Kestrel H', and 'Pablo H'. However, it is difficult to select the best varieties because each variety has its own advantages and disadvantages, and each variety needs to be selected for a particular product or specific function. The optimal composition of the controlled atmosphere storage for beetroots is $5 \% \mathrm{CO}_{2}$, $5 \% \mathrm{O}_{2}$ and $90 \% \mathrm{~N}_{2}$. It increases up to $10 \%$ of standard production compared to storage in simple storage chambers.

\section{References}

1. AOAC. (1990) Vitamin C (ascorbic acid) in vitamin preparations and juice. Helrich K. (ed.) Official Methods of Analysis, 15 ${ }^{\text {th }}$ edn, AOAC Inc., Arlington, VA, p. 1058.
2. AOAC. $\left(1990^{\mathrm{b}}\right)$ Sucrose in fruits and fruit products. Helrich K. (ed.), Official Methods of Analysis. 15 ${ }^{\text {th }}$ end, AOAC Inc., Arlington, VA, 922 p.

3. Badelek E., Adamicki F., Elkner K. (2002) The effect of temperature, cultivar and root size on quality and storage ability of red beet. Vegetable Crops Research Bulletin, Vol. 56, p. 67-76.

4. Bundinienè O., Viškelis P., Zalatorius V. (2007) Papildomo tręšimo per lapus ịtaka raudonųjų burokèlių derliui ir šakniavaisių kokybei. Sodininkyste ir daržininkystè, Vol. 26(1), p. 108-118.

5. Bundinienè O., Zalatorius V., Starkutè R. (2017) Naujos kartos trąšų ịtaka perdirbti skirtų burokèlių derliui ir jo kokybès rodikliams. Sodininkystė ir daržininkysté, Vol. 36(2-4), p. 36-46.

6. Cizauskas A., Viskelis P. (2002) Influence of nitrogenous fertilizers on onion yield, quality, and storability. In: Plant Nutrition: Growth and Diagnosis. Editors R. Dris, F.H. Abdelaziz, S.M. Jain. Science Publishers, Inc., Enfield, USA, Plymouth, UK, p. 129-135.

7. Esatbeyoglu T., Wagner A.E., Schini-Kerth V.B., Rimbach G. (2015) Betanin-A food colorant with biological activity. Molecular Nutrition and Food Research, Vol. 59, p. 36-47.

8. Gilchrist M., Winyard P.G., Fulford J., Annin, C., Shore A.C., Benjamin N. (2014) Dietary nitrate supplementation improves reaction time in type 2 diabetes: Development and application of a novel nitrate-depleted beetroot juice placebo. Nitric Oxide, Vol. 40, p. 67-74.

9. Henze J., Baumann. H. (1979) Quality of red beet (Beta vulgaris L.) as affected by storage conditions. Acta Horticulturae, Vol. 93, p. 59-66.

10. Lepse L., Viskelis P., Lepsis J., Bimsteine G. (2014) Influence of controlled atmosphere on the carrot storage quality. Acta Horticulturae, Vol. 1033, p. 59-64.

11. Luksiene Z., Buchovec I., Viskelis P. (2013) Impact of high-power pulsed light on microbial contamination, health promoting components and shelf life of strawberries. Food Technology and Biotechnology, Vol. 51(2), p. 284-292.

12. Methodological instructions for the determination of nitrates in crop production. (In Lithuanian). (1990) Vilnius, $41 \mathrm{p}$.

13. Moding K.J., Ferrante M.J., Bellows L.L., Bakke A.J., Hayes J.E., Johnson S.L. (2018) Variety and content of commercial infant and toddler vegetable products manufactured and sold in the United States. American Journal of Clinical Nutrition, Vol. 107, p. 576-583.

14. Petronienè O.D., Viškelis P. (2004) Biochemical composition and preservation of various red beet cultivars. Sodininkystè ir daržininkystè, Vol. 23(3), p. 89-97.

15. Simson S. P. Straus M. C. (2010) Post-harvest technology of horticultural crops. Oxford, $315 \mathrm{p}$.

16. Thompson A. K. Controlled atmosphere storage of fruits and vegetables, 2nd edition. CABI, 2010, $272 \mathrm{p}$.

17. Tucker W.G., Ward C.M., Davies A.C. (1977) An assessment of the long-term storage methods for beetroot, Acta Horticulturae, Vol. 62, p. 169-180.

18. Urbonavičienė D., Bobinaitė R., Trumbeckaitė S., Raudonè L., Janulis V., Bobinas Č., Viškelis P. (2018). Agro-industrial tomato by-products and extraction of functional food ingredients. Zemdirbyste-Agriculture, Vol. 105(2), p. 63-70.

19. Viškelienė A., Samuolienė G., Karklelienė R., ViškelisP., Sasnauskas A., Duchovskis P. (2017) Quality and developmental changes in white head cabbage (Brassica oleracea L.) and radish (Raphanus sativus L.) during 
winter storage. Zemdirbyste-Agriculture, Vol. 104(3), p. 229-234

20. Viškelis P., Bobinaite R., Lepse L., Lepsis J., Viškelis J. (2012) Quality changes of green onions stored in modified atmosphere. Sodininkyste ir daržininkystè, Vol. 31(1-2), p. 40-49.

21. Viskelis P., Rubinskienė M.,

Bobinaitè R., Dambrauskienè E. (2010) Bioactive compounds and antioxidant activity of small fruits in Lithuania. Journal of Food, Agriculture and Environment, Vol. 8(3-4), p. $259-263$.

22. Wroblewska M., JuskiewiczJ., Wiczkowski W. (2011) Physiological properties of beetroot crisps applied in standard and dyslipidaemic diets of rats. Lipids in Health and Disease, Vol. 10, p. 178-185.
23. Wruss J., Waldenberger G., Huemer S., Uygun P., Lanzerstorfer P., Müller U., Hoglinger O., Weghuber, J. (2015) Compositional characteristics of commercial beetroot products and beetroot juice prepared from seven beetroot varieties grown in Upper Austria. Journal of Food Composition and Analysis, Vol. 42, p. 46-55.

24. Zalatorius V., Bundinienè O., Kavaliauskaitė D. (2014) Intensyvi valgomujjų burokèlių auginimo technologija. Naujausios rekomendacijos žemès ir mišku ükiui. Akademija, Kèdainių, p. 59-64.

25. Ермаков А.И., Арасимович В.В., Ярош Н.П., Перуанский Ю.В., Луковникова Г.А., Иконникова М.И. (1987) Методы биохимического исследования растений, ВО Агропромиздат, $431 \mathrm{p}$. 